\title{
IMPLEMENTATION OF SINGLE-INDUCTOR DC/DC CONVERTER WITH FULLY RECONFIGURABLE STRUCTURE FOR RENEWABLE ENERGY APPLICATIONS
}

\author{
Pavithra.R, Mohamed Imran Khan S, Nishanth R, Niveth A, \\ Dept of EEE, RMK Engineering College
}

\begin{abstract}
:
A novel non-isolated three-port converter (NITPC) is used in this project. The purpose of this topology is to integrate a renewable energy and DC bus, instead of the widely reported consuming load, with a photovoltaic (PV)-battery system. Conventional methods require either a separate dc-dc converter to process the reversible power flow or employing an isolated three-port converter (TPC) which allows bi-directional power flow between any two ports. However, these methods require many switches which increases the converter size and control complexity. This project presents a compact but fully functional design by combining and integrating basic converters to form a simplified single inductor converter structure while keeping minimum number of switches. The resultant converter is fully reconfigurable that all possible power flow combinations among the sources and load are achieved through different switching patterns. In existing system, the single source (PV) will be used. So, the unidirectional power flow only possible. But In our proposed system Hybrid energy will be used. So, if any case in the system will be stable.
\end{abstract}

Keywords - Three port converter, Bi-directional Power flow, Single inductor converter structure.

\section{INTRODUCTION}

Recently, three-port converters are gaining popularity among applications which integrate renewable energies and energy storage with the load (e.g., satellite system, hybrid vehicle, etc.). A number of three-port converter (TPC) topologies have been proposed for the benefits of singlestage power conversion between any two ports. The formation of non-isolated TPCs (NITPCs) is based on a generic multiple-input multiple-output structure [1]- [3]. Zhang et al. [4] reviewed and compared recently published non-isolated, partly isolated and isolated TPCs. NITPCs are cost-effective and are compact due to the reduced components. Their control strategy is simple as NITPCs are derived from basic converters [2], [3]. However, for the same reason, the voltage gain of NITPC is limited. Partly isolated TPCs can provide a high voltage gain and isolation between two ports. However, they have limited power flow options due to partially shared devices. Isolated TPCs can achieve high voltage gain, high power operation and provide galvanic isolation while offering single-stage power conversion between any two ports. However, the three-windingtransformer and many switches increase the converter size and cost. Overall, most of the reviewed TPCs in [4] are designed for power consuming loads.

\section{EXISTING SYSTEM}

Block Diagram:

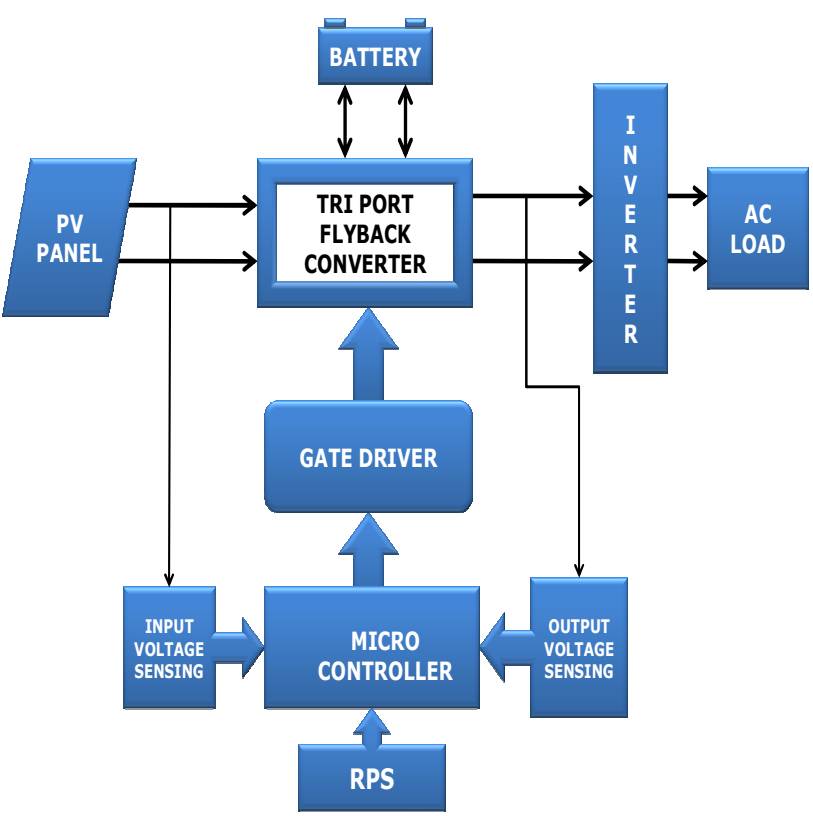


In the existing system TriPort Fly back converter is used which usually works in four modes. They are as follows:

- $\quad$ PV to DC Load

- $\quad$ PV to DC load \& Battery

- $\quad$ PV \& Battery to DC Load

- Battery to DC load

It follows Fly Back topology. The Coupled inductors are used to transfer the energy.

\section{Circuit Diagram:}

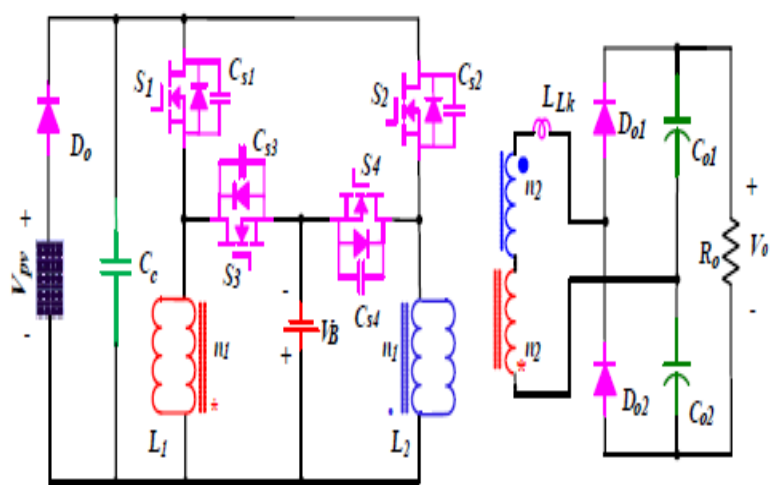

\section{Drawbacks in Existing System:}

- In this system only, PV energy will be utilized.

- Unidirectional conversion.

- More inductive loss will be high.

- Coupled inductors are used the transfer the energy.

\section{PROPOSED SYSTEM}

\section{Block Diagram:}

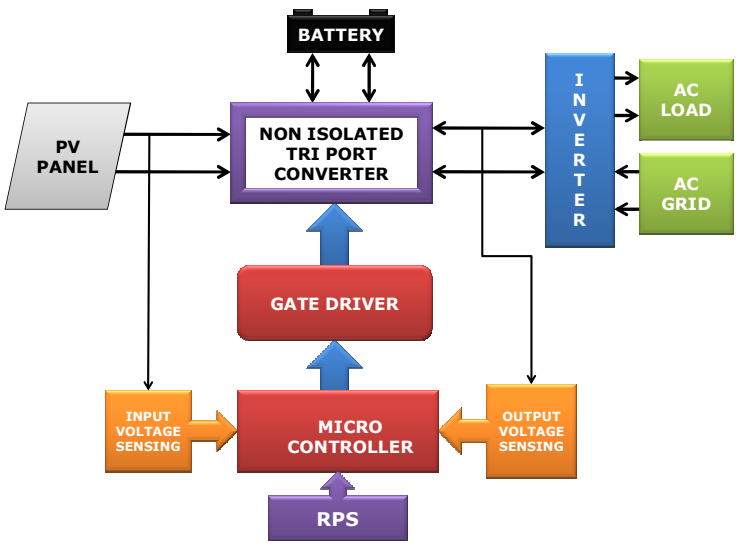

Solar and AC grid are used at the same time.The NonIsolated Tri port converter topology is proposed.Bi directional flow also possible.The Seven modes of conversion also possible. They are

- $\quad$ PV to DC grid.

- $\quad$ PV to DC grid and Battery.

- $\quad$ PV and Battery to DC grid.

- Battery to DC grid.

- PV to Battery.

- $\quad$ PV and DC grid to battery.

- DC grid to battery.

According to voltage difference the converter automatically select the mode.Single inverter is proposed to boost the voltage.

\section{Advantages of Proposed System:}

- Hybrid energy will be used.

- The system will be stable in all the conditions.

- The bidirectional flow will be achieved in this converter.

- Low inductive loss.

\section{Circuit Diagram:}

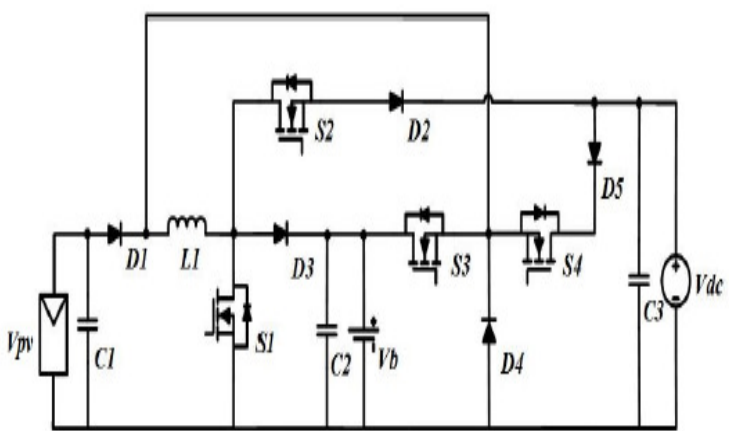

\section{Modes of Operation:}

\section{Mode 1: PV to Battery}

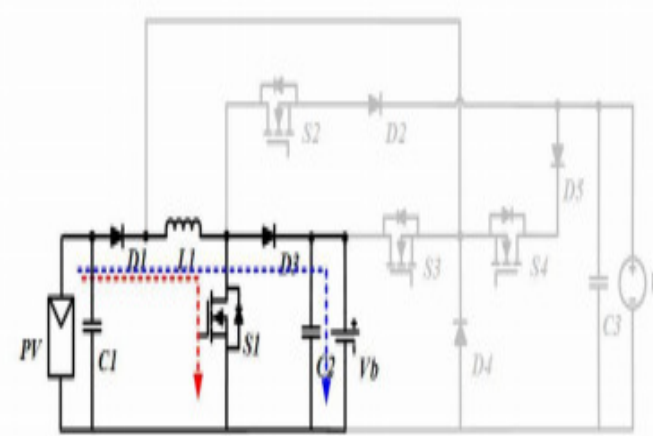

Mode 2: PV to Load 


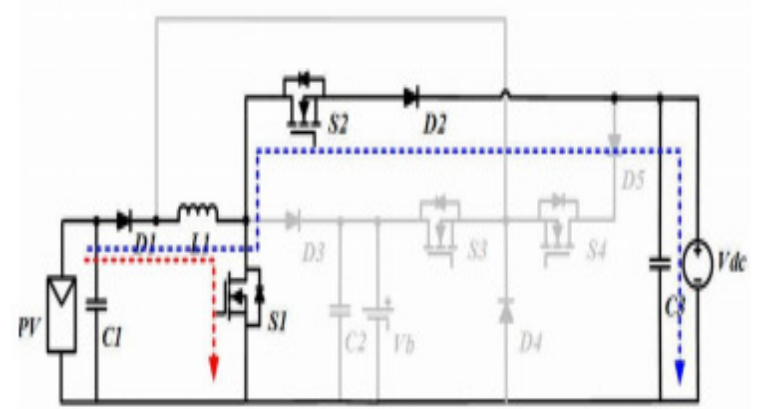

Mode 3: PV to Battery \& Load

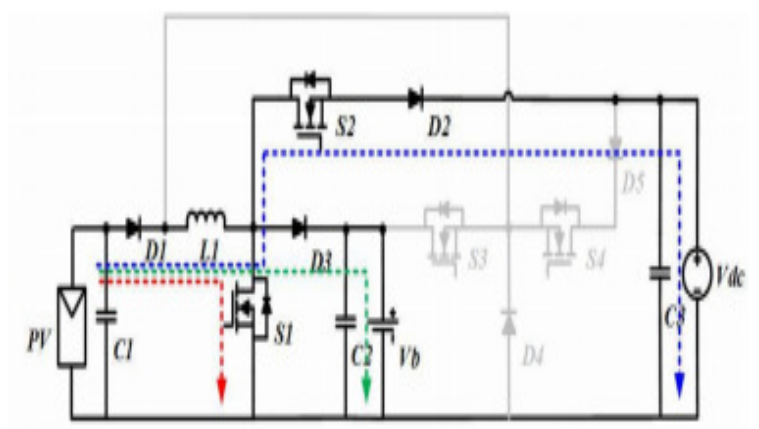

Mode 4: AC Source to Battery \& Load

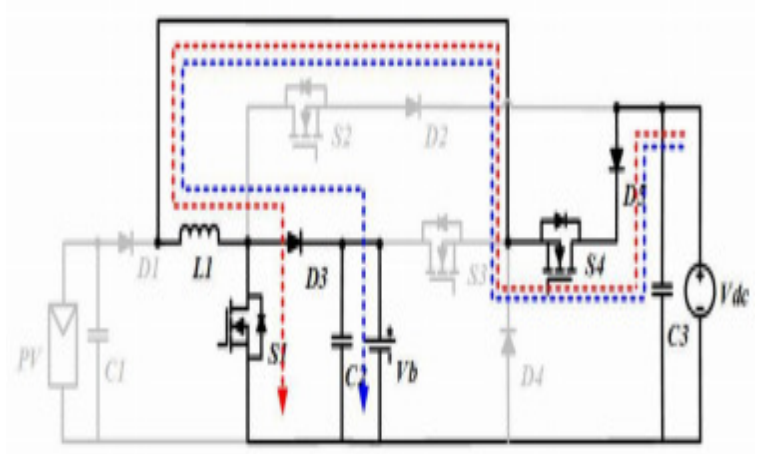

Mode 5: PV \& Battery to Load

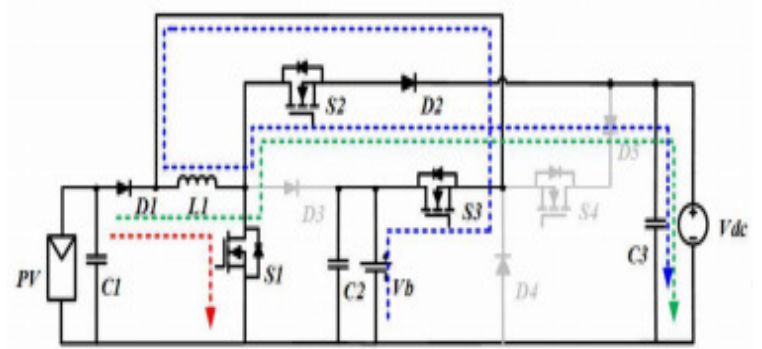

Mode 6: Battery to Load

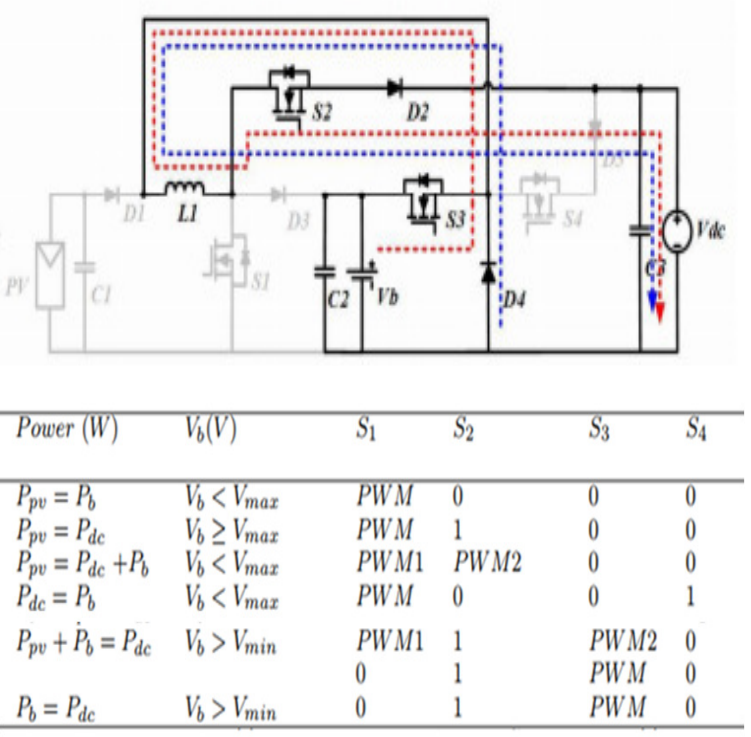

\section{CONVERTERS}

\section{BUCK CONVERTER(DC-DC):}

- A buck converter (dc-dc) is shown in Fig. Only a switch is shown, for which a device as described earlier belonging to transistor family is used.

- Also, a diode (termed as freewheeling) is used to allow the load current to flow through it, when the switch (i.e., a device) is turned off. The load is inductive (R-L) one. In some cases, a battery (or back EMF) is connected in series with the load (inductive).

- Due to the load inductance, the load current must be allowed a path, which is provided by the diode; otherwise, i.e., in the absence of the above diode, the high induced EMF of the inductance, as the load current tends to decrease, may cause damage to the switching device.

If the switching device used is a thyristor, this circuit is called as a step-down chopper, as the output voltage is normally lower than the input voltage. Similarly, this $\mathrm{dc}-\mathrm{dc}$ converter is termed as buck one, due to reason given later. 


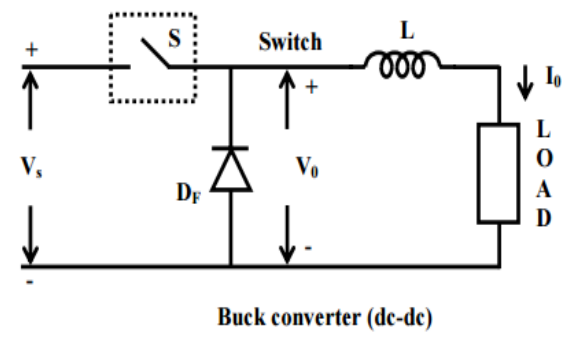

BOOST CONVERTER(DC-DC):

- Boost Converters (dc-dc) A boost converter (dcdc) is shown in fig. Only a switch is shown, for which a device belonging to transistor family is generally used.

- Also, a diode is used in series with the load. The load is of the same type as given earlier. The inductance of the load is small. An inductance, $\mathrm{L}$ is assumed in series with the input supply.

- The position of the switch and diode in this circuit may be noted, as compared to their position in the buck converter.

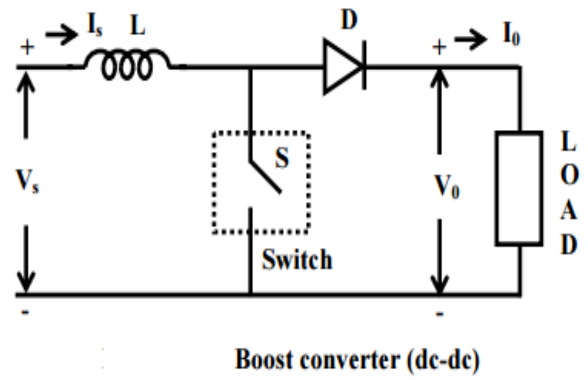

\section{BUCK-BOOST CONVERTERS (DC-DC)}

- A buck-boost converter (dc-dc) is shown in fig. Only a switch is shown, for which a device belonging to transistor family is generally used. Also, a diode is used in series with the load. The connection of the diode may be noted, as compared with its connection in a boost converter.

- The inductor, $\mathrm{L}$ is connected in parallel after the switch and before the diode. The load is of the same type as given earlier. A capacitor, $\mathrm{C}$ is connected in parallel with the load.

- The polarity of the output voltage is opposite to that of input voltage here. When the switch, $\mathrm{S}$ is put $\mathrm{ON}$, the supply current flows through the path, $\mathrm{S}$ and $\mathrm{L}$, during the time interval.

The currents through both source and inductor increase and are same, with being positive. The polarity of the induced voltage is same as that of the input voltage.

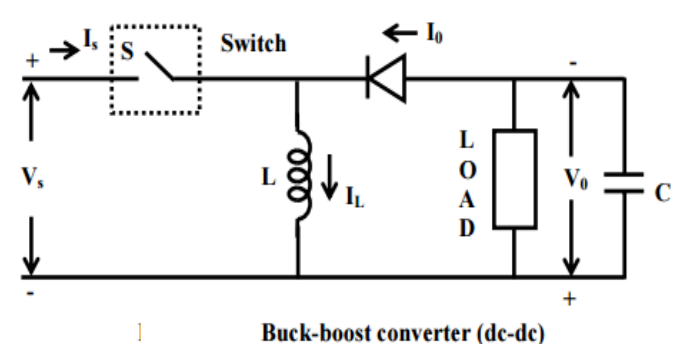

\section{THREE PORT CONVERTERS:}

Non-isolated Three-Port (DC/DC) converters that is NITPC intermeddling PV array, the battery and the load. Non-isolated converters contain an inductor instead of a transformer (isolated). Power efficiency of all three ports power flow paths in the Boost (DC/DC) TPCs is higher.As long as the Sun irradiation is high that is power produced by PV panel is greater than that of load demand.

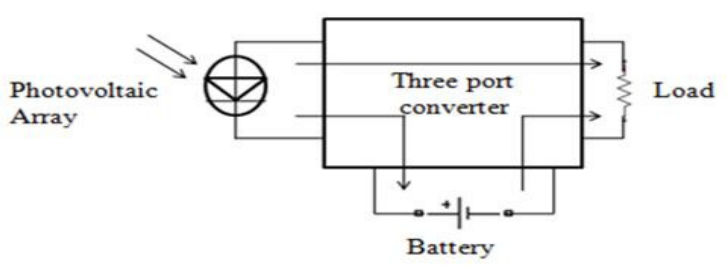

PV array feeds the load at the same time battery charges. When sun irradiation is low, battery discharges to the load because PV panel doesn't have sufficient power to meet load demand.

\section{GATE DRIVER}

$>$ Gate driver is a power amplifier that accepts a low power input from a controller IC and produces the appropriate high current gate drive for a power MOSFET.

$>$ They can also be integrated within a controller

$>$ The main purpose of gate driver circuit is 1. Isolation purpose

2. Efficient driving (PUSH-PULL amplifier)

3.PWM protector

\section{TLP 250}

Schematic Diagram:

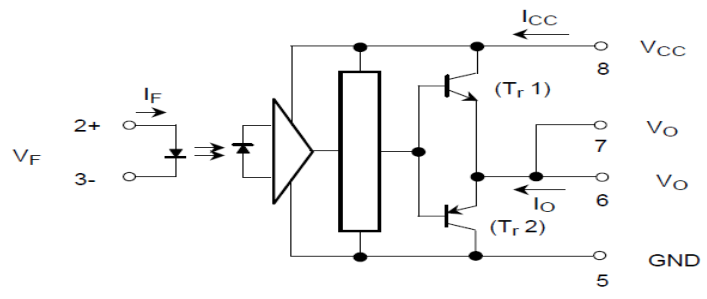




\section{Pin Diagram:}

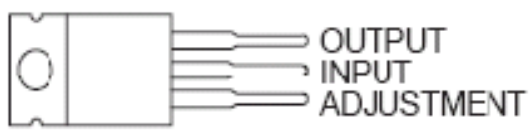

\section{TLP 250 IC Pin Diagram:}

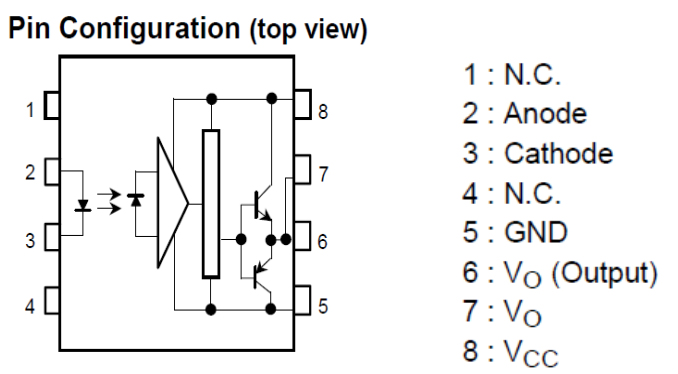

\section{POWER SUPPLY UNIT}

\section{BLOCK DIAGRAM}

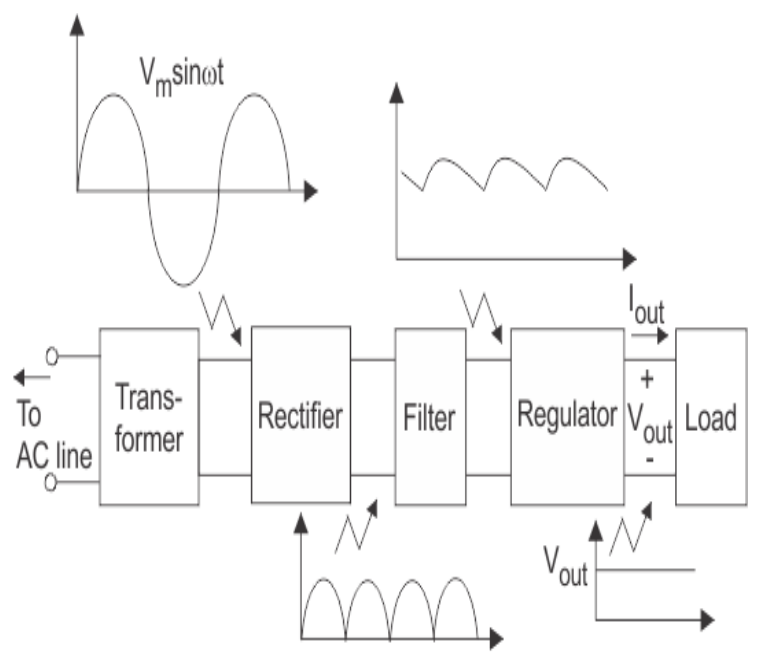

Components of typical linear power supply
As we all know any invention of latest technology cannot be activated without the source of power. So, it this fastmoving world we deliberately need a proper power source which will be apt for a particular requirement. All the electronic components starting from diode to Intel IC's only work with a DC supply ranging from $-+5 \mathrm{v}$ to $-+12 \mathrm{v}$. We are utilizing for the same, the cheapest and commonly available energy source of $230 \mathrm{v}-50 \mathrm{~Hz}$ and stepping down, rectifying, filtering and regulating the voltage. This will be dealt briefly in the forth-coming sections.

\section{STEP DOWN TRANSFORMER:}

When AC is applied to the primary winding of the power transformer it can either be stepped down or up depending on the value of DC needed. In our circuit the transformer of $230 \mathrm{v} / 15-0-15 \mathrm{v}$ is used to perform the step-down operation where a $230 \mathrm{~V}$ AC appears as $15 \mathrm{~V}$ AC across the secondary winding. One alteration of input causes the top of the transformer to be positive and the bottom negative. The next alteration will temporarily cause the reverse. The current rating of the transformer used in our project is $2 \mathrm{~A}$. Apart from stepping down AC voltages, it gives isolation between the power source and power supply circuitries.

\section{RECTIFIER UNIT:}

In the power supply unit, rectification is normally achieved using a solid-state diode. Diode has the property that will let the electron flow easily in one direction at proper biasing condition. As AC is applied to the diode, electrons only flow when the anode and cathode is negative. Reversing the polarity of voltage will not permit electron flow.A commonly used circuit for supplying large amounts of DC power is the bridge rectifier. A bridge rectifier of four diodes $(4 *$ IN4007) are used to achieve full wave rectification. Two diodes will conduct during the negative cycle and the other two will conduct during the positive half cycle. The DC voltage appearing across the output terminals of the bridge rectifier will be somewhat less than $90 \%$ of the applied RMS value. Normally one alteration of the input voltage will reverse the polarities. Opposite ends of the transformer will therefore always be 180 degrees out of phase with each other.

\section{FILTERING UNIT:}

Filter circuits which are usually capacitors acting as a surge arrester always follow the rectifier unit. This capacitor is also called as a decoupling capacitor or a bypassing capacitor, is used not only to 'short' the ripple with frequency of $120 \mathrm{~Hz}$ to ground but also to leave the frequency of the DC to appear at the output. A load resistor $\mathrm{R} 1$ is connected so that a reference to the ground is maintained. C1R1 is for bypassing ripples. $\mathrm{C} 2 \mathrm{R} 2$ is used as a low pass filter, i.e. it passes only low frequency signals 
and bypasses high frequency signals. The load resistor should be $1 \%$ to $2.5 \%$ of the load.

$1000 \propto \mathrm{f} / 25 \mathrm{v} \quad$ : for the reduction of ripples from the pulsating.

$10 \propto \mathrm{f} / 25 \mathrm{v} \quad$ : for maintaining the stability of the voltage at the load side.

$\mathrm{O}, 1 \propto \mathrm{f} \quad$ : for bypassing the high frequency disturbances.

\section{Dual Regulated Power Supply:}

DC SIGNAL CONDITIONING:

Signal Conditioning Unit:

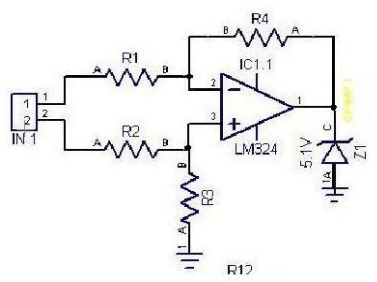

Signal conditioning can include amplification, filtering, converting, range matching, isolation and any other processes required to make sensed output suitable for processing after rectifying.

\section{Voltage Sensing -Potential Divider:}

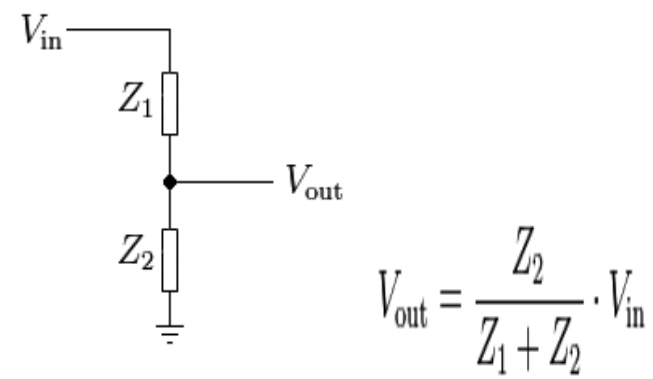

A voltage divider (also known as a potential divider) is a linear circuit that produces an output voltage (Vout) that is a fraction of its input voltage (Vin). Voltage division refers to the partitioning of a voltage among the components of the divider.

\section{Current Sensing-Current Divider:}

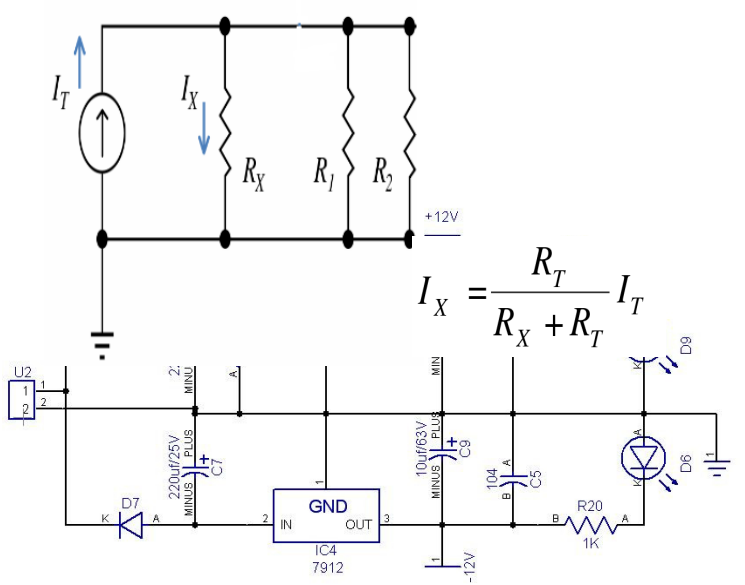

Current division refers to the splitting of current between the branches of the divider.The currents in the various branches of such a circuit will always divide in such a way as to minimize the total energy expended.

\section{PIC MICROCONTROLLER}

The microcontroller is the heart of the power saving unit, which get the data from sensor and driver the control circuit. It is an integrated chip that is often part of an embedded system. The microcontroller includes a $\mathrm{CPU}$ RAM, ROM, I/O ports and timers like a standard computer but they are designed to execute only a single specific task to control a single system they are much smaller and simplified so that they can include all the functions required on a single chip. Other than the normal Microcontrollers PIC Family supports more features.

- PIC microcontroller is widely used for experimental and modern applications because of its low price, wide range of applications, high quality and ease of availability. It is ideal for machine control applications, measurement devices, and study purpose and so on.

- It is also called as "Computer on a Chip".

- $\quad$ PIC was developed as Peripheral controller.

- PIC Microcontrollers are designed with a separate 14-bit program memory bus to carry instructions.

- A Separate 8bit data memory bus to carry data.

- This Design is commonly called Harvardarchitecture, and So PIC Microcontroller is based on Harvard architecture. 
- Every instruction is coded as a single 14-bitword and fetched simultaneously with the corresponding data variable for that instruction.

- The Harvard architecture speeds up the Process by its design.

- The instruction set for the PIC Microcontroller consist of 35 instructions.

- $\quad$ Each Occupying a single 14-bit program memory word and a two stage Pipelining.

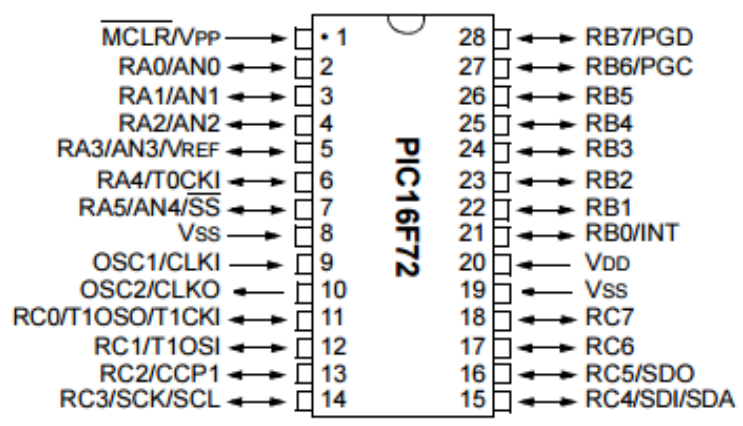

It consists of Flash Memory which make the programming cost and time leSS

$>$ PIC come with one of four CPU cores

$>$ 12-bitcores with 33 instructions: $12 \mathrm{C} 50 \mathrm{x}, 16 \mathrm{C} 5 \mathrm{x}$

$>$ 14-bitcores with 35 instructions: $12 \mathrm{C} 50 \mathrm{x}, 16 \mathrm{C} 5 \mathrm{x}$

$>$ 16-bitcores with 58 instructions: $12 \mathrm{C} 50 \mathrm{x}, 16 \mathrm{C} 5 \mathrm{x}$

$>$ Enhanced 16-bitcores with 77 instructions: $18 \mathrm{CXX}$

Special Microcontroller Features:

- 1,000 erase/write cycle FLASH program memory typical

- Power-on Reset (POR), Power-up Timer (PWRT) and

Oscillator Start-up Timer (OST)

- Watchdog Timer (WDT) with its own on-chip RC oscillator for reliable operation

- Programmable code protection

- Power saving SLEEP mode
- Selectable oscillator options

- In-Circuit Serial Programming ${ }^{\text {TM }}$ (ICSPTM) via 2 pins

Peripheral Features:

- High Sink/Source Current: $25 \mathrm{~mA}$

- Timer0: 8-bit timer/counter with 8-bit pre-scalar

- Timer1: 16-bit timer/counter with pre-scalar, can be incremented during SLEEP via external crystal/clock

- Timer2: 8-bit timer/counter with 8-bit period register, prescalar and post scaler

- Capture, Compare, PWM (CCP) module

$>$ Capture is 16-bit, max. resolution is $12.5 \mathrm{~ns}$

$>$ Compare is 16-bit, max. resolution is $200 \mathrm{~ns}$

$>$ PWM max. resolution is 10-bit

- 8-bit, 5-channel analog-to-digital converter

- Synchronous Serial Port (SSP) with SPI ${ }^{\mathrm{TM}}$

(Master/Slave) and I2CTM (Slave)

- Brown-out detection circuitry for Brown-out Reset (BOR)

Reason for choosing PIC16F72

- Low power, high speed CMOS FLASH technology

- Fully static design

- Wide operating voltage range: $2.0 \mathrm{~V}$ to $5.5 \mathrm{~V}$

- Industrial temperature range

- Low power consumption: - < 0.6 mA typical @ 3V, 4 $\mathrm{MHz}-20 \mu \mathrm{A}$ typical @ 3V, $32 \mathrm{kHz}-<1 \mu \mathrm{A}$ typical standby current

\section{SOFTWARE IMPLEMENTATION AND SIMULATION RESULTS}

\section{PROTEUS:}

Proteus (PROcessor for TExt Easy to USe) is a fully functional, procedural programming language created in 1998 by Simone Zanella. Proteus incorporates many functions derived from several other

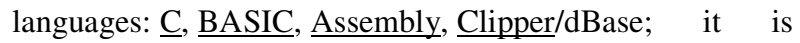
especially versatile in dealing with strings, having hundreds of dedicated functions; this makes it one of the richest languages for text manipulation.

\section{Introduction}


Proteus is a high-performance simulator for MIMD multiprocessors. It is fast, accurate, and flexible: it is one to two orders of magnitude faster than comparable simulators,
- comprehensibility of Proteus scripts;

- availability of advanced data structures: arrays, queues (single or double), stacks, bit

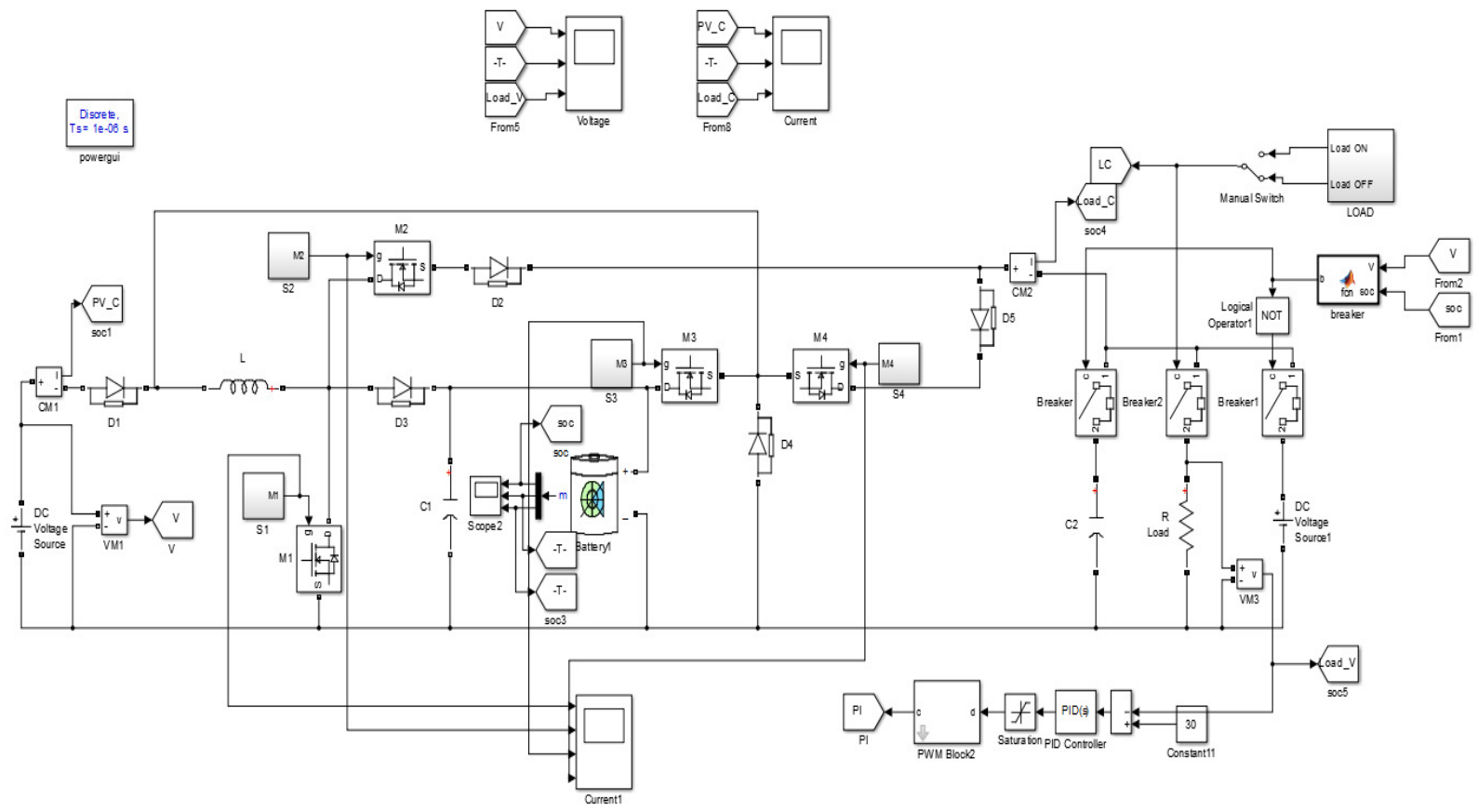

it can reproduce results from real multiprocessors, and it is easily configured to simulate a wide range of architectures. Proteus providesa modular structure that simplifies customization and independent replacement of parts of architecture. There are typically multiple implementations of each module that provide different combinations of accuracy and performance; users pay for accuracy only when and where they need it. Finally, Proteus provides repeatability, nonintrusive monitoring and debugging, and integrated graphical output, whichresult in a development environment superior to those available on real multiprocessors.Proteus was initially created as a multiplatform (DOS, Windows, $\underline{\text { Unix }}$ ) system utility, to manipulate text and binary files and to create CGI scripts. The language was later focused on Windows, by adding hundreds of specialized functions for: network and serial communication, database interrogation, system service creation, console applications, keyboard emulation, ISAPI scripting (for IIS). Most of these additional functions are only available in the Windows flavor of the interpreter, even though a Linux version is still available.

Proteus was designed to be practical (easy to use, efficient, complete), readable and consistent.

Its strongest points are:

- $\quad$ powerful string manipulation. maps, sets, AVL trees.

The language can be extended by adding user functions written in Proteus or DLLs created in $\mathrm{C} / \mathrm{C}++$.

\section{Features:}

$\square$ Proteus has a fully functional, procedural approach;

$\square$ Variables are untyped, do not need to be declared, can be local or public and can be passed by value or by reference; $\square$ All the typical control structures are available (if-thenelse; for-next; while-loop; repeat-until; switch-case);

$\square$ New functions can be defined and used as native functions.

Data types supported by Proteus are only three: integer numbers, floating point numbers and strings. Access to advanced data structures (files, arrays, queues, stacks, AVL trees, sets and so on) takes place by using handles, i.e. integer numbers returned by item creation functions. Type declaration is unnecessary: variable type is determined by the function applied - Proteus converts on the fly every variable when needed and holds previous data renderings, to avoid performance degradation caused by repeated conversions. There is no need to add parenthesis in expressions to determine the evaluation order, because the language is fully functional (there are no operators). 
Proteus includes hundreds of functions for:

$\square$ Accessing file system.

$\square$ Sorting data.

Manipulating dates and strings.

$\square$ Interacting with the user (console functions)

$\square$ Calculating logical and mathematical expressions.

\section{SIMULATION CIRCUIT:}

\section{SIMULATION OUTPUT:}

\section{Load OFF Condition(Current):}

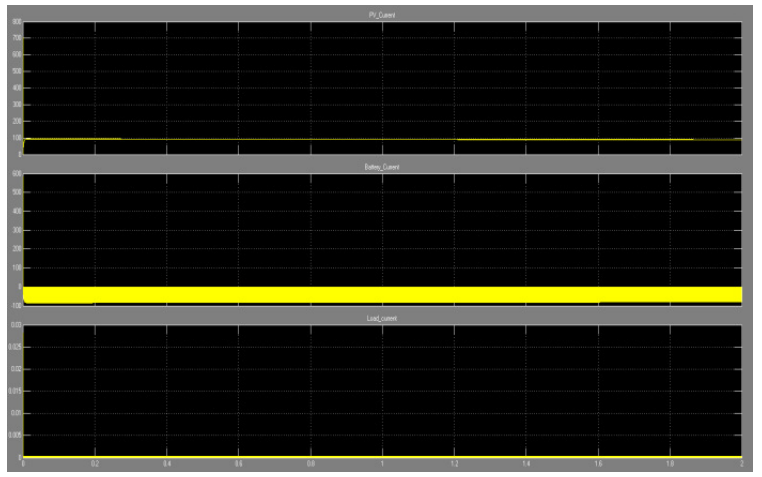

Load OFF Condition(Voltage):

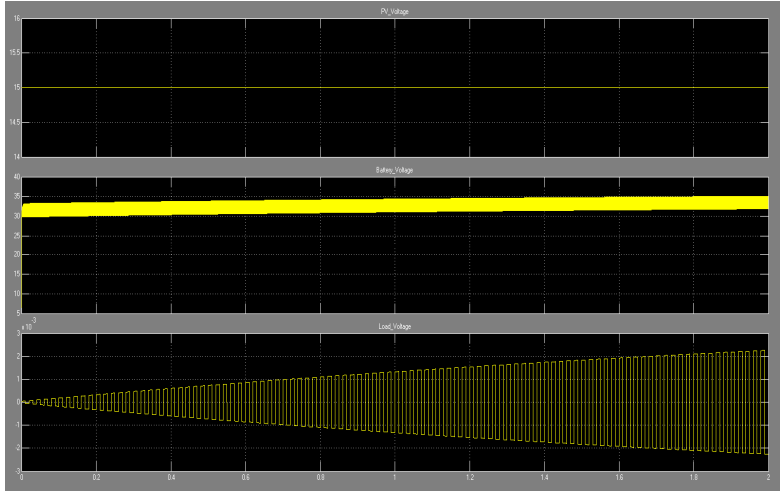

Load OFF Condition(Switch Status):

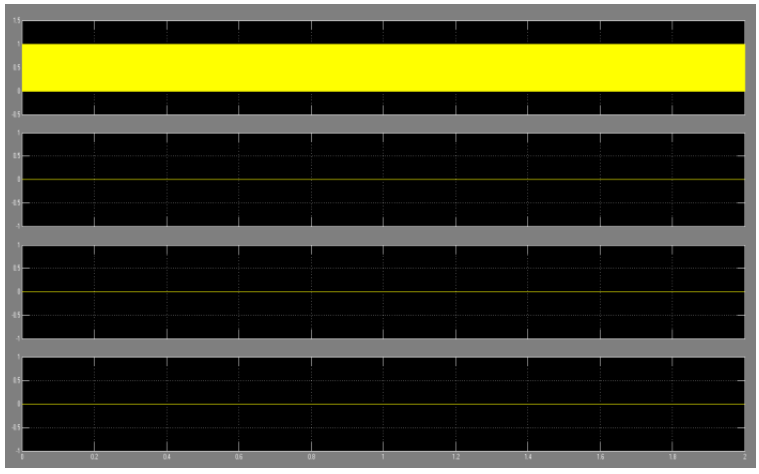

\section{Switch Status:}

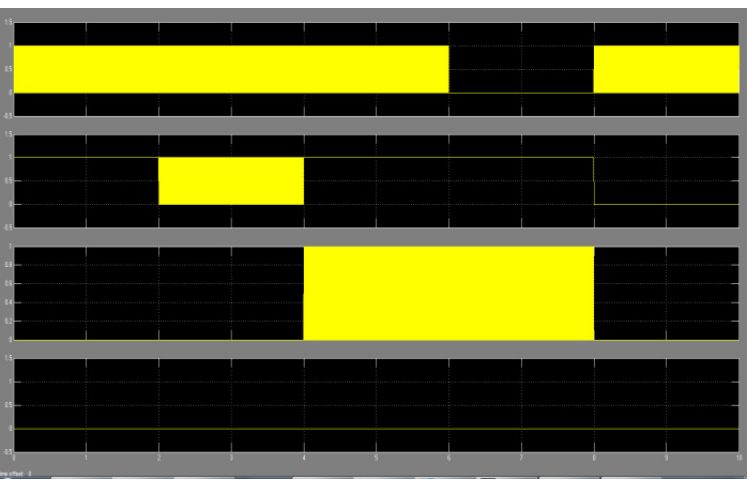

Voltage Level:

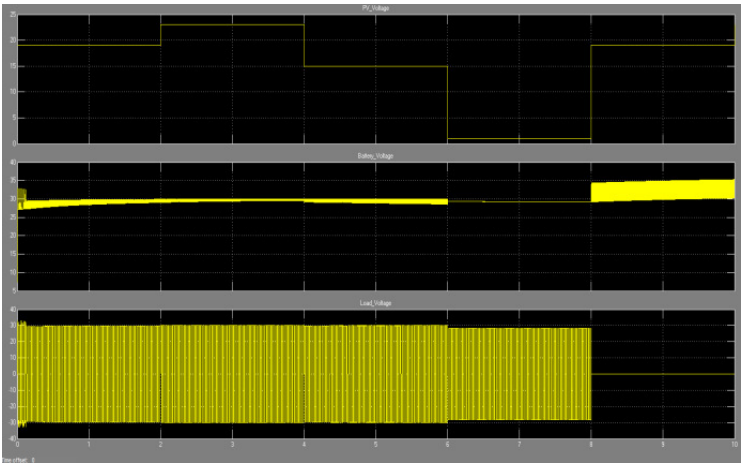

Current Level:

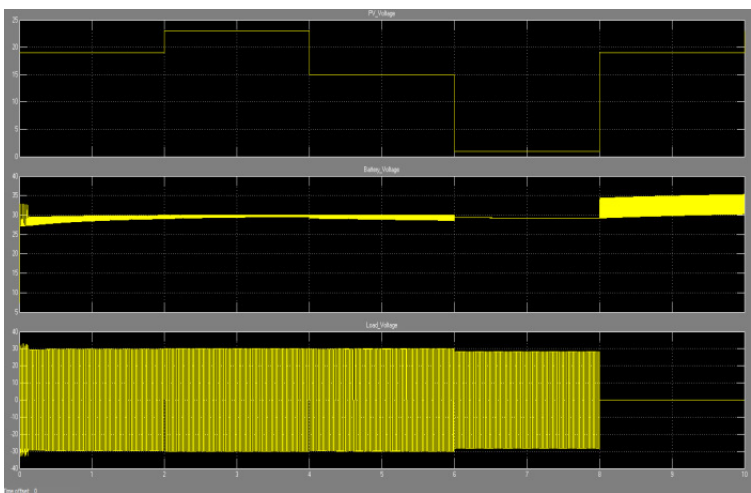




\section{CONCLUSION}

The purpose of this project is to integrate a renewable energy and DC bus, instead of the widely reported consuming load, with a photovoltaic (PV)-battery system. This project replaces theconventional method which require either a separate dc-dc converter to process the reversible power flow or employing an isolated three-port converter (TPC) which allows bi-directional power flow between any two ports. This project presents a compact but fully functional design by combining and integrating basic converters to form a simplified single inductor converter structure while keeping minimum number of switches. The resultant converter is fully reconfigurable that all possible power flow combinations among the sources and load are achieved through different switching patterns. The proposedHybrid energy system will be stable for any case.

\section{REFERENCES:}

[1] Dongwon Kwon, Gabriel A. Rincón-Mora,2009,SingleInductor-Multiple-Output Switching DC-DC Converters. [2]Young-Jin Moon, Yong-SeongRoh, Jung-Chul Gong, and Changsik Yoo,2012,Load-Independent Current Control Technique of a Single-Inductor Multiple-Output Switching DC-DC converter.

[3]Kyoung-SikSeol, Young-Jin Woo, Gyu-Hyeong Cho, Gyu-Ha Gho,2009,A Synchronous Multioutput Step Up/Down DC-DC Converter With Return Current Control. [4]Ghulam Fareed Laghari,Adnan Uma, Suheel Abdullah,2015,Comparative Analysis of Multi-Input DC/DC Converter Topology for Hybrid Renewable Energy Systems.

[5]XiaolingXiong,Chi K. Tse,

XinboRuan,2013,Bifurcation Analysis of StandalonePhotovoltaicBattery Hybrid Power System. [6]Muhammad Aamir, Saad Mekhilef,2015,High Gain Zero Voltage Switching Bidirectional converter with reduced number of switches 